\title{
Error Estimates for the Heterogeneous Multiscale Finite Volume Method of Convection-Diffusion-Reaction Problem
}

\author{
Tao Yu $\mathbb{D}^{1},{ }^{1}$ Peichang Ouyang $\mathbb{D},{ }^{1}$ and Haitao Cao $\mathbb{D}^{2}$ \\ ${ }^{1}$ Department of Mathematics and Physics, Jinggangshan University, Ji'an 343009, China \\ ${ }^{2}$ Department of Mathematics and Physics, Hohai University, Changzhou Campus, Changzhou 213022, China
}

Correspondence should be addressed to Peichang Ouyang; g_fcayang@163.com

Received 10 May 2018; Accepted 10 September 2018; Published 8 October 2018

Academic Editor: Yan-Ling Wei

Copyright (C) 2018 Tao Yu et al. This is an open access article distributed under the Creative Commons Attribution License, which permits unrestricted use, distribution, and reproduction in any medium, provided the original work is properly cited.

Based on the heterogeneous multiscale method, this paper presents a finite volume method to solve multiscale convectiondiffusion-reaction problem. The paper constructs an algorithm of the optimal order convergence rate in $H^{1}$-norm under periodic medias.

\section{Introduction}

This paper considers the multiscale method for the following convection-diffusion-reaction problem

$$
\left\{\begin{array}{l}
-\nabla\left(a^{\varepsilon}(x) \nabla u^{\varepsilon}(x)\right)+b^{\varepsilon}(x) \nabla u^{\varepsilon}(x)+c^{\varepsilon}(x) u^{\varepsilon}(x)=f(x), \quad x \in \Omega, \\
u^{\varepsilon}(x)=0, \quad x \in \partial \Omega
\end{array}\right.
$$

where $\Omega \subset \mathbb{R}^{2}$ (or $\mathbb{R}^{3}$ ) is a bounded convex polygonal domain with Lipschitz boundary $\partial \Omega, \varepsilon \ll 1$ is a positive parameter which signifies the multiscale nature of (1). This problem is related to groundwater and solute transport in porous media [1].

Optimal order convergence rate of classical finite element method based on piecewise linear polynomials relies on the $H^{2}$-norm of $u^{\varepsilon}$. As coefficients vary on a scale of $\varepsilon$, the solution $u^{\varepsilon}$ may also oscillate at the same scale. A direct numerical solution of this multiscale problem is very difficult to derive unless the mesh size is sufficiently smaller. However, it is not feasible in practice since the amount of computation will increase sharply as the amount of calculation increases. On the other hand, from an engineering point of view, the macroscopic features of the solution are often of main interest and importance. According to homogenization theory [2, 3], there is a homogenized equation which can capture the macroscopic properties. In other words, there exist homogenized coefficients $a^{0}, b^{0}$, and $c^{0}$ so that

$$
\left\{\begin{array}{l}
-\nabla\left(a^{0}(x) \nabla u^{0}(x)\right)+b^{0}(x) \nabla u^{0}(x)+c^{0}(x) u^{0}(x)=f(x), \quad x \in \Omega, \\
u^{0}(x)=0, \quad x \in \Omega .
\end{array}\right.
$$

Though there exist many classical methods for solving (2), unfortunately, in general, there are no explicit formulae for the homogenized coefficients, except that there are many restrictive assumptions on the media. Thus, developing numerical methods that can capture the effect of small scale on the large scale is an attractive subject. To overcome the above difficulties, many strategies have been established to solve the problems on grids which are coarser than the scale of oscillation; see [4-9] and references therein.

The multiscale finite volume (MSFV) method was first introduced by Jenny et al. for the elliptic problem of highly oscillatory coefficients [9]. Based on a special interpolation from the coarse mesh to the fine mesh, this method captured the effect of small scales on a coarse grid efficiently. It not only fitted into finite volume framework nicely but also kept both the conservation of coarse and fine scales. This method was widely applied in many situations, such as discrete 


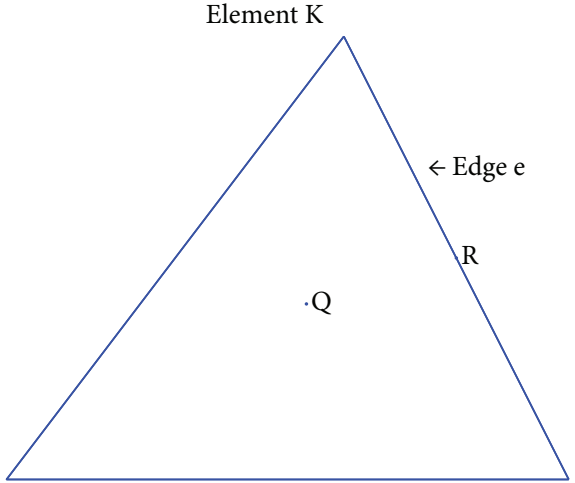

(a)

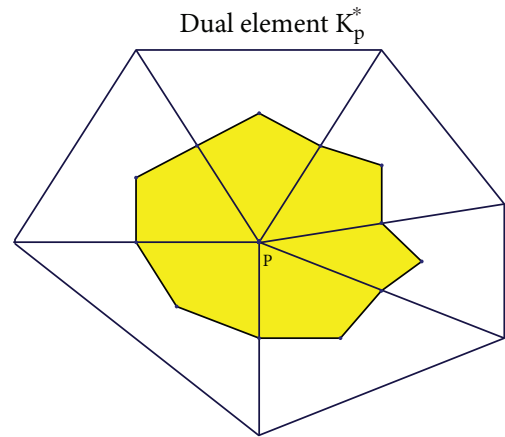

(b)

Figure 1: An element $K$ (a) and its dual element (b) with respect to a node $P$.

fracture modeling [10], parabolic problem [11], and Maxwell's equations [12]. Recently, Weinan E. and Engquist established a general efficient methodology-heterogeneous multiscale method (HMM) [13], for the multiscale problems, which consists of two components: (a) selecting a macroscopic solver on a coarse mesh and (b) estimating the missing macroscale data by solving local fine-scale problems. The careful selection of the macro solver and local fine problems is a key issue for the method. A different choice of macroscopic solver will lead to a different heterogeneous multiscale method; see some examples for finite element method $[8,14,15]$ and discontinuous finite element method $[4,16]$. It is well known that the finite volume method has many advantages, such as keeping conservation and applying to complex regions. Thus, in this paper, we choose the finite volume method (FVM) introduced in [17] as the macroscopic solver. For convenience, the heterogeneous multiscale method taking finite volume method as the macroscopic solver is denoted as HMM-FVM. We will show that our method has optimal order convergence rate in $H^{1}$-norm for periodic medias.

The rest of this paper is organized as follows. To solve (1), Section 2 first constructs a HMM-FVM. Then, Section 3 will study the approximate solution and its error estimates for periodic media associated with (1) in detail. We finally conclude the paper in Section 4.

\section{HMM-FVM for Convection-Diffusion- Reaction Problem}

In this section, we first concisely describe the finite volume method for convection-diffusion-reaction problem in [17]. Then, using the method of [17] as a macroscopic solver of HMM, we derive the HMM-FVM in detail.

Let $\mathscr{T}_{H}$ be a quasi-uniform triangulation of the polygonal domain $\Omega$. The barycenter dual decomposition $\mathscr{T}_{H}^{*}$ is constructed by connecting the barycenter to the midpoints of edges of each triangle element. Suppose $K$ is a triangle element, denote $e$ and $Q$, respectively, as an edge and barycenter of $K$ and $R$ the midpoint of $e$. Assume $P$ is a nodal point and $K_{P}^{*}$ is the dual element with respect to
$P$ (referring to Figure 1). Let $H$ be the maximum length of the edges, $N_{H}$ the set of all nodal points, $\dot{K}$ the vertices of $K$.

Let $V=H_{0}^{1}(\Omega)=\left\{u \in H^{1}(\Omega) \mid u=0\right.$ on $\left.\partial \Omega\right\}$ and $V_{H} \subset V$ be the piecewise linear finite element space on $\mathscr{T}_{H}, \Pi_{H}^{*}$ be the interpolation operator from $V_{H}$ to the piecewise constant space on $\mathscr{T}_{H}^{*}$ :

$$
\left(\Pi_{H}^{*} v_{H}\right)(x)=v_{H}(P), x \in K_{P}^{*}, \forall P \in N_{H}, v_{H} \in V_{H} .
$$

Then, the finite volume method [17] reads as finding $u_{H} \in V_{H}$ such that

$$
A\left(u_{H}, \Pi_{H}^{*} v_{H}\right)=\left(f, \Pi_{H}^{*} v_{H}\right), \forall v_{H} \in V_{H},
$$

where

$$
\begin{aligned}
A\left(u_{H}, \Pi_{H}^{*} v_{H}\right)= & \sum_{P \in N_{H}}\left[-\int_{\partial K_{P}^{*}} \mathbf{n} \cdot\left(a^{H} \nabla u_{H}\right) \Pi_{H}^{*} v_{H} d s\right. \\
& \left.+\int_{K_{P}^{*}}\left(b^{H} \nabla u_{H}+c^{H} u_{H}\right) \Pi_{H}^{*} v_{H} d x\right] \\
= & \sum_{K \in \mathscr{T}_{H}}\left[-\sum_{P \in K} \int_{K \cap \partial K_{P}^{*}} \mathbf{n} \cdot\left(a^{H} \nabla u_{H}\right) \Pi_{H}^{*} v_{H} d s\right. \\
& \left.+\int_{K}\left(b^{H} \nabla u_{H}+c^{H} u_{H}\right) \Pi_{H}^{*} v_{H} d x\right], \\
\left(f, \Pi_{H}^{*} v_{H}\right)= & \sum_{P \in N_{H}} \int_{K_{P}^{*}} f \Pi_{H}^{*} v_{H} d x=\sum_{K \in \mathscr{T}_{H}} \int_{K} f \Pi_{H}^{*} v_{H} d x .
\end{aligned}
$$

We next construct our multiscale method with respect to (1). 
By the numerical integration for the barycenter quadrature rule, (5) and (6) can be written as

$$
\begin{aligned}
A_{F V M}\left(u_{H}, \Pi_{H}^{*} v_{H}\right)= & \sum_{K \in \mathscr{T}_{H}}\left[-\sum_{P \in \dot{K}}\left|K \cap \partial K_{P}^{*}\right| \mathbf{n}\right. \\
& \cdot\left(a^{H}(Q) \nabla u_{H}\right) \Pi_{H}^{*} v_{H}+|K|\left(b^{H}(Q) \nabla u_{H}\right. \\
& \left.\left.+c^{H}(Q) u_{H}(Q)\right) \Pi_{H}^{*} v_{H}\right],
\end{aligned}
$$$$
\left(f, \Pi_{H}^{*} v_{H}\right)_{H}=\sum_{K \in \mathscr{T}_{H}}|K| f(Q) \Pi_{H}^{*} v_{H} .
$$

Thus, the barycenter quadrature approximation of the FVM can be read as finding $u_{H} \in V_{H}$ such that

$$
A_{F V M}\left(u_{H}, \Pi_{H}^{*} v_{H}\right)=\left(f, \Pi_{H}^{*} v_{H}\right)_{H}, \forall v_{H} \in V_{H} .
$$

To obtain error estimate, this paper considers the coefficients of scale separation. Assume that the multiscale coefficients $a^{\varepsilon}(x), b^{\varepsilon}(x)$, and $c^{\varepsilon}(x)$ have the forms $a(x, x / \varepsilon)$, $b(x, x / \varepsilon)$, and $c(x, x / \varepsilon)$. Furthermore, assume that $a(x, y), b$ $(x, y)$, and $c(x, y)$ are smooth in $x$ and periodic in $y$ with respect to the unit cube $Y$.

In the absence of explicit expressions of $a^{H}, b^{H}$, and $c^{H}$, $a^{H}(Q) \nabla u_{H}, b^{H}(Q) \nabla u_{H}$, and $c^{H}(Q)$ can be approximated by

$$
\begin{aligned}
a^{H}(Q) \nabla u_{H} & \simeq \frac{1}{\left|K_{\delta}(Q)\right|} \int_{K_{\delta}(Q)} a\left(Q, \frac{x}{\varepsilon}\right) \nabla R\left(u_{H}\right) d x, \\
b^{\mathrm{H}}(Q) \nabla u_{H} & \simeq \frac{1}{\left|K_{\delta}(Q)\right|} \int_{K_{\delta}(Q)} b\left(Q, \frac{x}{\varepsilon}\right) \nabla R\left(u_{H}\right) d x, \\
c^{H}(Q) & \simeq \frac{1}{\left|K_{\delta}(Q)\right|} \int_{K_{\delta}(Q)} c\left(Q, \frac{x}{\varepsilon}\right) d x,
\end{aligned}
$$

where $R\left(u_{H}\right)$ is the solution of the following microcell problem

$$
\left\{\begin{array}{l}
-\nabla \cdot\left(a\left(Q, \frac{x}{\varepsilon}\right) \nabla R\left(u_{H}\right)\right)=0 \text { in } K_{\delta}(Q), \\
R\left(u_{H}\right)=u_{H}, \text { on } \partial K_{\delta}(Q),
\end{array}\right.
$$

and $K_{\delta}(Q)$ is a cube of size $\delta$ centered at $Q$.

Thus, the HMM-FVM can be summarized as the following variational problem: $\forall v_{H} \in V_{\mathrm{H}}$, find $u_{H} \in V_{H}$ satisfying (9)-(11).

\section{A Priori Error Estimate}

This section deduces the main result of the paper-optimal $H^{1}$ error estimate (Theorem 2). To this end, we first introduce Lemma 1 and Lemma 2 to derive its modeling error. Then, by regarding the HMM-FVM as a perturbation of the linear finite element method (Theorem 1), we obtain the optimal error estimate of HMM-FVM.

Lemma $1([14,18,19])$. There exists a constant $C$ independent of $H, \varepsilon$, and $\delta$ such that for $a^{H}, b^{H}$,

$$
\begin{aligned}
& \left|a_{i j}^{H}-a_{i j}^{0}\right| \leq C \frac{\varepsilon}{\delta}, \quad i, j=1,2, \\
& \left|b_{i}^{H}-b_{i}^{0}\right| \leq C \frac{\varepsilon}{\delta}, \quad i=1,2,
\end{aligned}
$$

where $a_{i j}^{0}=1 /|Y| \int_{Y} a_{i k}(x, y)\left(\delta_{k j}+\left(\partial x^{j} / \partial y_{k}\right)(x, y)\right) d y \quad b_{i}^{0}=1 /$ $|Y| \int_{Y}\left(b_{i}(x, y)+a_{i k}\left(\partial \eta / \partial y_{k}\right)(x, y)\right) d y$. Here, $\chi^{j}$ and $\eta$ are, respectively, the periodic solutions of $-\nabla_{y} \cdot\left(a(x, y) \nabla_{y} \chi^{j}(x, y)\right)$ $=\nabla_{y} \cdot\left(a(x, y) e_{j}\right),-\nabla_{y} \cdot\left(a(x, y) \nabla_{y} \eta(x, y)\right)=\nabla_{y} \cdot b(x, y)$ with zero mean (i.e., $\int_{Y} \chi^{j} d y=0, \int_{Y} \eta d y=0$ ).

Lemma $2([18,20])$. Given domain $K_{\delta}$ with $\operatorname{diam}(K)=\delta$, let $\varphi(s, y)$ defined in $Y$ be a $Y$-periodic function in $y$, where $Y$ is a unit cube and $s \in R$ is fixed. Then

$$
\left|\frac{1}{|Y|} \int_{Y} \varphi(s, y) d y-<\varphi\left(s, \frac{x}{\varepsilon}\right)>_{K}\right| \leq \mathrm{C} \frac{\varepsilon}{\delta},
$$

where $C$ is independent of $\varepsilon, \delta$, and $s$.

Mathematically, the finite element method for the homogenization problem (2) is equivalent to finding $u_{H} \epsilon$ $V_{H}$ such that

$$
B\left(u_{H}, v_{H}\right)=\left(f, v_{H}\right), \forall v_{H} \in V_{H},
$$

where

$$
\begin{aligned}
B\left(u_{H}, v_{H}\right) & =\sum_{K \in \mathscr{T}_{H}} \int_{K}\left(a^{0} \nabla u_{H} \cdot \nabla v_{H}+b^{0} \nabla u_{H} v_{H}+c^{0} u_{H} v_{H}\right) d x \\
\left(f, v_{H}\right) & =\sum_{K \in \mathscr{T}_{H}} \int_{K} f v_{H} d x .
\end{aligned}
$$

Applying barycenter quadrature to (17), the bilinear form can be refined as

$$
\begin{aligned}
\tilde{B}\left(u_{H}, v_{H}\right)= & \sum_{K \in \mathscr{T}_{H}}|K|\left(a^{0}(Q) \nabla u_{H} \bullet \nabla v_{H}+b^{0}(Q) \nabla u_{H} v_{H}(Q)\right. \\
& \left.+c^{0}(Q) u_{H}(Q) v_{H}(Q)\right) .
\end{aligned}
$$

Finally, to estimate the priori error estimate, we borrow inf-sup condition of bilinear form $B$ given in [17]. 
Lemma 3 [17]. For $0<H<h_{0}$,

$$
\left\|u_{H}\right\|_{1, \Omega} \leq C \sup _{0 \neq v_{H} \in V_{H}} \frac{B\left(u_{H}, v_{H}\right)}{\left\|v_{H}\right\|_{1, \Omega}}, \forall u_{H} \in V_{H} .
$$

The following theorem characterizes the difference between the bilinear form of HMM-FVM and FEM, which will play the key role in the subsequent analysis.

Theorem 1. $\forall u_{H}, v_{H} \in V_{H}$, we have

$$
\left|B\left(u_{H}, v_{H}\right)-A_{F V M}\left(u_{H}, v_{H}\right)\right| \leq C\left(H+\frac{\varepsilon}{\delta}\right)\left\|u_{H}\right\|_{1, \Omega}\left\|v_{H}\right\|_{1, \Omega},
$$

where $C$ is a positive constant independent of $\varepsilon, \delta$, and $H$.

Proof. The bilinear form can be split into three terms

$$
\begin{aligned}
& \left|B\left(u_{H}, v_{H}\right)-A_{F V M}\left(u_{H}, v_{H}\right)\right| \leq\left|B\left(u_{H}, v_{H}\right)-\tilde{B}\left(u_{H}, v_{H}\right)\right| \\
& \quad+\left|\tilde{B}\left(u_{H}, v_{H}\right)-\tilde{A}\left(u_{H}, v_{H}\right)\right|+\mid \tilde{A}\left(u_{H}, v_{H}\right) \\
& \quad-A_{F V M}\left(u_{H}, v_{H}\right) \mid:=\varepsilon_{1}\left(u_{H}, v_{H}\right)+\varepsilon_{2}\left(u_{H}, v_{H}\right) \\
& \quad+\varepsilon_{3}\left(u_{H}, v_{H}\right),
\end{aligned}
$$

where

$$
\begin{aligned}
\tilde{A}\left(u_{H}, v_{H}\right)= & \sum_{K \in \mathscr{T}_{H}}|K|\left(a^{H}(Q) \nabla u_{H} \cdot \nabla v_{H}+b^{H}(Q) \nabla u_{H} v_{H}(Q)\right. \\
& \left.+c^{H}(Q) u_{H}(Q) v_{H}(Q)\right) .
\end{aligned}
$$

Denote by

$$
E_{K}(f)=\int_{K} f(x) d x-|K| f(Q) .
$$

By the standard estimate of [21], we get

$$
\left|E_{K}(a(x) p(x) q(x))\right| \leq C H\|a\|_{1, \infty}\|p\|_{0, K}\|q\|_{0, K} .
$$

Accordingly, the numerical quadrature error $\varepsilon_{1}\left(u_{H}\right.$, $\left.v_{H}\right)$ is

$$
\begin{aligned}
\varepsilon_{1}\left(u_{H}, v_{H}\right) \leq & \sum_{K \in \mathscr{T}_{H}}\left(\left|E_{K}\left(a^{0} \nabla u_{H} \cdot \nabla v_{H}\right)\right|+\left|E_{K}\left(b^{0} u_{H} \nabla v_{H}\right)\right|\right. \\
& \left.+\left|E_{K}\left(c^{0} u_{H} v_{H}\right)\right|\right) \leq C H\left\|u_{H}\right\|_{1, \Omega}\left\|v_{H}\right\|_{1, \Omega} .
\end{aligned}
$$

We next utilize Lemma 1 and Lemma 2 to estimate the modeling error $\varepsilon_{2}\left(u_{H}, v_{H}\right)$. By the definition of $c^{H}$ in (11) and Lemma 2, we see that the error between $c^{H}$ and $c^{0}$ can be treated as

$$
\left|c^{H}-c^{0}\right| \leq C \frac{\varepsilon}{\delta},
$$

where $c^{0}=1 /|Y| \int_{Y} c(x, y) d y$ and $C$ is a positive constant independent of $\varepsilon, \delta$, and $H$. Let

$$
e(H M M)=\max _{x \in \Omega}\left\{\left|a_{i j}^{H}-a_{i j}^{0}\right|,\left|b_{i}^{H}-b_{i}^{0}\right|,\left|c^{H}-c^{0}\right|\right\} .
$$

By Lemma 1 and (27),

$$
e(H M M) \leq C \frac{\varepsilon}{\delta} .
$$

Thus, the modeling error $\varepsilon_{2}\left(u_{H}, v_{H}\right)$ can be further estimated as

$$
\begin{aligned}
\varepsilon_{2}\left(u_{H}, v_{H}\right) & \leq C e(H M M)\left\|u_{H}\right\|_{1, \Omega}\left\|v_{H}\right\|_{1, \Omega} \\
& \leq C \frac{\varepsilon}{\delta}\left\|u_{H}\right\|_{1, \Omega}\left\|v_{H}\right\|_{1, \Omega} .
\end{aligned}
$$

It remains to estimate the term $\varepsilon_{3}\left(u_{H}, v_{H}\right)$ which concerns three components. We next start this work.

By Green's formula, the first term of $\tilde{A}\left(u_{H}, v_{H}\right)$ is

$$
\begin{aligned}
|K| a^{H}(Q) \nabla u_{H} \nabla v_{H}= & \int_{K} a^{H}(Q) \nabla u_{H} \nabla v_{H} d x \\
= & -\int_{K} \nabla\left(a^{H}(Q) \nabla u_{H}\right) v_{H} d x \\
& +\int_{\partial K}^{n} n \cdot\left(a^{H}(Q) \nabla u_{H}\right) v_{H} d s \\
= & \int_{\partial K} n \cdot\left(a^{H}(Q) \nabla u_{H}\right) v_{H} d s .
\end{aligned}
$$

The first term of $A_{F V M}\left(u_{H}, v_{H}\right)$ can be derived similarly

$$
\begin{aligned}
& -\sum_{P \in \dot{K}}\left|K \cap \partial K_{P}^{*}\right| n \cdot\left(a^{H}(Q) \nabla u_{H}\right) \Pi_{H}^{*} v_{H} \\
& =-\sum_{P \in \dot{K}} \int_{K \cap \partial K_{P}^{*}} n \cdot\left(a^{H}(Q) \nabla u_{H}\right) \Pi_{H}^{*} v_{H} d s \\
& =-\sum_{P \in \dot{K}} \int_{\partial\left(K \cap \partial K_{P}^{*}\right)} n \cdot\left(a^{H}(Q) \nabla u_{H}\right) \Pi_{H}^{*} v_{H} d s \\
& +\int_{\partial K} n \cdot\left(a^{H}(Q) \nabla u_{H}\right) \Pi_{H}^{*} v_{H} d s \\
& =-\int_{K} \nabla\left(a^{H}(Q) \nabla u_{H}\right) \Pi_{H}^{*} v_{H} d x \\
& +\int_{\partial K} n \cdot\left(a^{H}(Q) \nabla u_{H}\right) \Pi_{H}^{*} v_{H} d s \\
& =\int_{\partial K} n \cdot\left(a^{H}(Q) \nabla u_{H}\right) \Pi_{H}^{*} v_{H} d s .
\end{aligned}
$$


The combination of the two gives

$$
\begin{aligned}
& \sum_{K \in \mathscr{T}_{H}}|K| a^{H}(Q) \nabla u_{H} \nabla v_{H} \\
& -\sum_{K \in \mathscr{T}_{H}}\left(-\sum_{P \in \dot{K}}\left|K \cap \partial K_{P}^{*}\right| n \cdot\left(a^{H}(Q) \nabla u_{H}\right) \Pi_{H}^{*} v_{H}\right) \\
& \quad=\sum_{K \in \mathscr{T}_{H}}\left(\sum_{e \in \partial K} \int_{e} n \cdot\left(a^{H}(Q) \nabla u_{H}\right)\left(v_{H}-\Pi_{H}^{*} v_{H}\right) d s\right) .
\end{aligned}
$$

Recall the interpolation operator $\Pi_{H}^{*}$ (see Lemma 2.1 in [17]) satisfying

$$
\left\|v_{H}-\Pi_{H}^{*} v_{H}\right\|_{0, K} \leq C H\left|v_{H}\right|_{1, K}, \forall v_{H} \in V_{H} .
$$

From (29), (33), and (34), the last term $\varepsilon_{3}\left(u_{H}, v_{H}\right)$ can be estimated as

$$
\begin{aligned}
\varepsilon_{3}\left(u_{H}, v_{H}\right)= & \sum_{K \in \mathscr{T}_{H}}\left[\sum_{e \in \partial K} \int_{e} n \cdot\left(a^{H}(Q) \nabla u_{H}\right)\left(v_{H}-\Pi_{H}^{*} v_{H}\right) d s\right. \\
& \left.+|K|\left(b^{H}(Q) \nabla u_{H}+c^{H}(Q) u_{H}\right)\left(v_{H}-\Pi_{H}^{*} v_{H}\right)\right] \\
= & \sum_{K \in \mathscr{T}_{H}}|K|\left(b^{H}(Q) \nabla u_{H}+c^{H}(Q) u_{H}\right)\left(v_{H}-\Pi_{H}^{*} v_{H}\right) \\
\leq & \sum_{K \in \mathscr{T}_{H}}|K|\left(\left\|b^{H}\right\|_{0, \infty, K}\left\|\nabla u_{H}\right\|_{0, \infty, K}\right. \\
& \left.+\left\|c^{H}\right\|_{0, \infty, K}\left\|u_{H}\right\|_{0, \infty, K}\right)\left\|v_{H}-\Pi_{H}^{*} v_{H}\right\|_{0, \infty, K} \\
\leq & C \sum_{K \in \mathscr{T}_{H}}\left(\left\|b^{H}\right\|_{0, \infty, K}\left\|\nabla u_{H}\right\|_{0,2, K}\right. \\
& \left.+\left\|c^{H}\right\|_{0, \infty, K}\left\|u_{H}\right\|_{0,2, K}\right)\left\|v_{H}-\Pi_{H}^{*} v_{H}\right\|_{0,2, K} \\
\leq & C\left(\left\|b^{H}\right\|_{0, \infty, \Omega}+\left\|c^{H}\right\|_{0, \infty, \Omega}\right)\left\|u_{H}\right\|_{1, \Omega} \| v_{H} \\
& -\Pi_{H}^{*} v_{H} \|_{0, \Omega} \leq C H\left(\left\|b^{H}\right\|_{0, \infty, \Omega}+c^{H}{ }_{0, \infty, \Omega}\right) \\
& \cdot\left\|u_{H}\right\|_{1, \Omega}\left|v_{H}\right|_{1, \Omega} \leq C H\left(\left\|b^{0}\right\|_{0, \infty, \Omega}+\left\|c^{0}\right\|_{0, \infty, \Omega}\right. \\
& +e(H M M))\left\|u_{H}\right\|_{1, \Omega}\left|v_{H}\right|_{1, \Omega} \\
\leq & \left.\left.C(H+e(H M M))\left\|u_{H}\right\|_{1, \Omega}\right|_{H}\right|_{1, \Omega} \\
\leq & C\left(H+\frac{\varepsilon}{\delta}\right)\left\|u_{H}\right\|_{1, \Omega}\left|v_{H}\right|_{1, \Omega} \cdot
\end{aligned}
$$

By the estimates of $\varepsilon_{1}\left(u_{H}, v_{H}\right), \varepsilon_{2}\left(u_{H}, v_{H}\right)$, and $\varepsilon_{3}\left(u_{H}, v_{H}\right)$, we finally get

$$
\left|B\left(u_{H}, v_{H}\right)-A_{F V M}\left(u_{H}, v_{H}\right)\right| \leq C\left(H+\frac{\varepsilon}{\delta}\right)\left\|u_{H}\right\|_{1, \Omega}\left\|v_{H}\right\|_{1, \Omega} .
$$

With the preparation of Theorem 1, we summarize the main result in Theorem 2 .
Theorem 2. Denote by $u^{0}$ and $u_{H}$ the solution of (2) and (9), respectively. Then for sufficient small $H$ and $\varepsilon / \delta$, we have

$$
\left\|u^{0}-u_{H}\right\|_{1, \Omega} \leq C\left(H+\frac{\varepsilon}{\delta}\right)
$$

where $C$ is a positive constant independent of $\varepsilon, \delta$, and $H$.

Proof. In order to estimate the error between $u^{0}$ and $u^{H}$, we separate it into two parts

$$
\left\|u^{0}-u_{H}\right\|_{1, \Omega} \leq\left\|u^{0}-u_{H}^{0}\right\|_{1, \Omega}+\left\|u_{H}^{0}-u_{H}\right\|_{1, \Omega},
$$

where $u_{H}^{0}$ is the numerical solution of (16), which is FEM formula of the homogenized problem (2).

From the standard error estimate [21], the first part of (38) can be estimated as

$$
\left\|u^{0}-u_{H}^{0}\right\|_{1, \Omega} \leq C H\left\|u^{0}\right\|_{1, \Omega} .
$$

It remains to treat the second part of (38). By Lemma 3, we have

$$
\begin{aligned}
\left\|u_{H}^{0}-u_{H}\right\|_{1, \Omega} \leq & C \sup _{0 \neq v_{H} \in V_{H}} \frac{B\left(u_{H}^{0}-u_{H}, v_{H}\right)}{\left\|v_{H}\right\|_{1, \Omega}} \\
\leq & C \sup _{0 \neq v_{H} \in V_{H}}\left(\frac{\left|B\left(u_{H}, v_{H}\right)-A_{F V M}\left(u_{H}, \Pi_{H}^{*} v_{H}\right)\right|}{\left\|v_{H}\right\|_{1, \Omega}}\right. \\
& \left.+\frac{\left|\left(f, v_{H}\right)_{H}-\left(f, \Pi_{H}^{*} v_{H}\right)_{H}\right|}{\left\|v_{H}\right\|_{1, \Omega}}\right):=T_{1}+T_{2} .
\end{aligned}
$$

According to Theorem 1, $T_{1}$ can be estimated as

$$
\begin{aligned}
T_{1} & \leq C\left(H+\frac{\epsilon}{\delta}\right)\left\|u_{H}\right\|_{1, \Omega} \\
& \leq C\left(H+\frac{\epsilon}{\delta}\right)\left(\left\|u^{0}\right\|_{1, \Omega}+\left\|u_{H}-u^{0}\right\|_{1, \Omega}\right)
\end{aligned}
$$

On the other hand, the numerator of $T_{2}$ in (40) is

$$
\begin{aligned}
\left(f, v_{H}\right)_{H}-\left(f, \Pi_{H}^{*} v_{H}\right)_{H} & =\sum_{K} \int_{K} f v_{H} d x-\sum_{K}|K| f(Q) \Pi_{H}^{*} v_{H} \\
& =\sum_{K} \int_{K} f \mathrm{v}_{H} d x-\sum_{K} \int_{K} f(Q) \Pi_{H}^{*} v_{H} d x \\
& =\sum_{K} \int_{K} f v_{H} d x-\sum_{K} \int_{K} f(Q) v_{H} d x \\
& =\sum_{K} \int_{K}(f(x)-f(Q)) v_{H} d x \\
& \leq C H \sum_{K}\|\nabla f\|_{0,2, K}\left\|v_{H}\right\|_{0,2, K} \\
& \leq C H|f|_{1, \Omega}\left\|v_{H}\right\|_{0, \Omega} \\
& \leq C H|f|_{1, \Omega}\left\|v_{H}\right\|_{1, \Omega} .
\end{aligned}
$$


Thus,

$$
T_{2} \leq \mathrm{CH}|f|_{1, \Omega}
$$

We see (37) follows immediately by combining the results of (38)-(43).

\section{Conclusion}

This paper investigates the multiscale convection-diffusionreaction problem. Based on the heterogeneous multiscale method, we first choose a finite volume method as macroscopic solver elaborately which can keep conservation and can be applied to complex regions. Then, we construct a suitable HMM-FVM strategy to solve the problem. We show that this method possesses the optimal order convergence rate in $H^{1}$-norm for periodic medias. As a matter of fact, this method can be applied in practice to multiscale problems without assumptions of periodic and scale separation.

\section{Data Availability}

The data used to support the findings of this study are available from the corresponding author upon request.

\section{Conflicts of Interest}

The authors declare that they have no conflicts of interest.

\section{Acknowledgments}

This work was supported by the National Natural Science Foundation of China (nos. 11461035 and 11761039), Science and Technology Plan Project of Jiangxi Provincial Education Department (nos. GJJ160749 and GJJ160758) and the Doctoral Starting up Foundation of Jinggangshan University (no. JZB11002).

\section{References}

[1] J. Bear, Dynamics of Fluids in Porous Media, American Elsevier, New York, 1972.

[2] A. Bensoussan, J.-L. Lions, and G. Papanicolaou, Asymptotic Analysis for Periodic Structures, Studies in Mathematics and Its Applications, vol. 5, North-Holland Publ, New York, 1978.

[3] V. V. Jikov, S. M. Kozlov, and O. A. Oleinik, Homogenization of Differential Operators and Integral Functionals, SpringerVerlag, Berlin, 1994.

[4] A. Abdulle and M. E. Huber, "Discontinuous Galerkin finite element heterogeneous multiscale method for advectiondiffusion problems with multiple scales," Numerische Mathematik, vol. 126, no. 4, pp. 589-633, 2014.

[5] A. Abdulle and M. E. Huber, "Numerical homogenization method for parabolic advection-diffusion multiscale problems with large compressible flows," Numerische Mathematik, vol. 136, no. 3, pp. 603-649, 2017.

[6] F. Brezzi, D. Marini, and E. süli, "Residual-free bubbles for advection-diffusion problems: the general error analysis," Numerische Mathematik, vol. 85, no. 1, pp. 31-47, 2000.
[7] W. Deng, X. Yun, and C. Xie, "Convergence analysis of the multiscale method for a class of convection-diffusion equations with highly oscillating coefficients," Applied Numerical Mathematics, vol. 59, no. 7, pp. 1549-1567, 2009.

[8] P. Henning and M. Ohlberger, "The heterogeneous multiscale finite element method for advection-diffusion problems with rapidly oscillating coefficients and large expected drift," Networks and Heterogeneous Media, vol. 5, no. 4, pp. 711-744, 2010.

[9] P. Jenny, S. H. Lee, and H. A. Tchelepi, "Multi-scale finitevolume method for elliptic problems in subsurface flow simulation," Journal of Computational Physics, vol. 187, no. 1, pp. 47-67, 2003.

[10] S. Bosma, H. Hajibeygi, M. Tene, and H. A. Tchelepi, "Multiscale finite volume method for discrete fracture modeling on unstructured grids (MS-DFM)," Journal of Computational Physics, vol. 351, no. 15, pp. 145-164, 2017.

[11] H. Hajibeygi and P. Jenny, "Multiscale finite-volume method for parabolic problems arising from compressible multiphase flow in porous media," Journal of Computational Physics, vol. 228, no. 14, pp. 5129-5147, 2009.

[12] E. Haber and L. Ruthotto, "A multiscale finite volume method for Maxwell's equations at low frequencies," Geophysical Journal International, vol. 199, no. 2, pp. 1268-1277, 2014.

[13] E. Weinan and B. Engquist, "The heterogeneous multi-scale methods," Communications in Mathematical Sciences, vol. 1, pp. 87-132, 2003.

[14] E. Weinan, P. Ming, and P. Zhang, "Analysis of the heterogeneous multiscale method for elliptic homogenization problems," Journal of the American Mathematical Society, vol. 18, no. 1, pp. 121-157, 2005.

[15] P. Ming and P. Zhang, "Analysis of the heterogeneous multiscale method for parabolic homogenization problems," Mathematics of Computation, vol. 76, no. 257, pp. 153-178, 2007.

[16] A. Abdulle, "Discontinuous Galerkin finite element heterogeneous multiscale method for elliptic problems with multiple scales," Mathematics of Computation, vol. 81, no. 278, pp. 687-713, 2012.

[17] H. Wu and R. Li, "Error estimates for finite volume element methods for general second-order elliptic problems," Numerical Methods for Partial Differential Equations, vol. 19, no. 6, pp. 693-708, 2003.

[18] H. Ye, W. Deng, and Z. Chen, "A new upscaling method for the solute transport equations," Discrete and Continuous Dynamical Systems, vol. 13, no. 4, pp. 941-960, 2005.

[19] X. H. Wu, T. Hou, and Y. Efendiev, "Analysis of upscaling absolute permeability," Discrete and Continuous Dynamical Systems - Series B, vol. 2, no. 2, pp. 185-204, 2002.

[20] Z. Chen, W. Deng, and H. Ye, "Upscaling of a class of nonlinear parabolic equations for the flow transport in heterogeneous porous media," Communications in Mathematical Sciences, vol. 3, no. 4, pp. 493-515, 2005.

[21] P. G. Clarlet, The Finite Element Method for Elliptic Problems, North-Holland Publishing Company, North-Holland, Amsterdam, New York, 1978. 


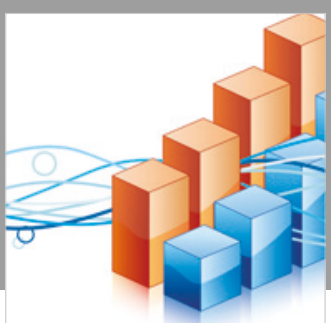

Advances in

Operations Research

\section{-n-m}
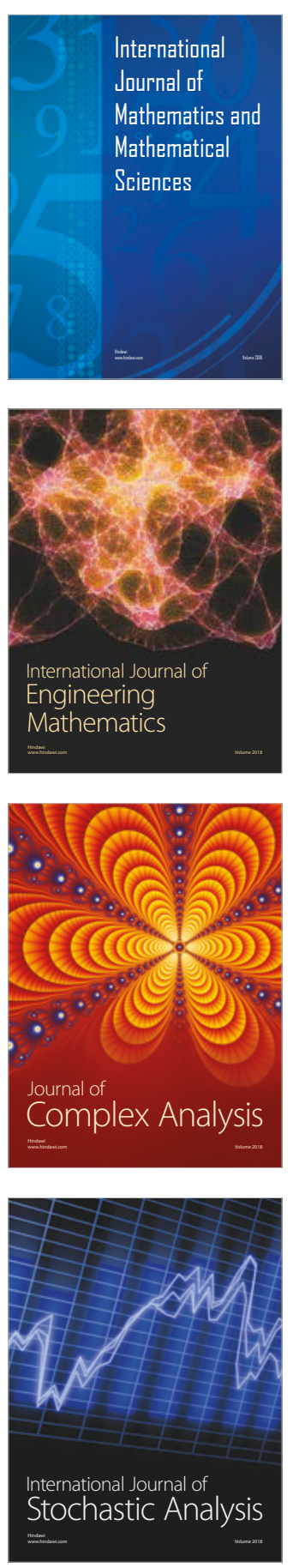
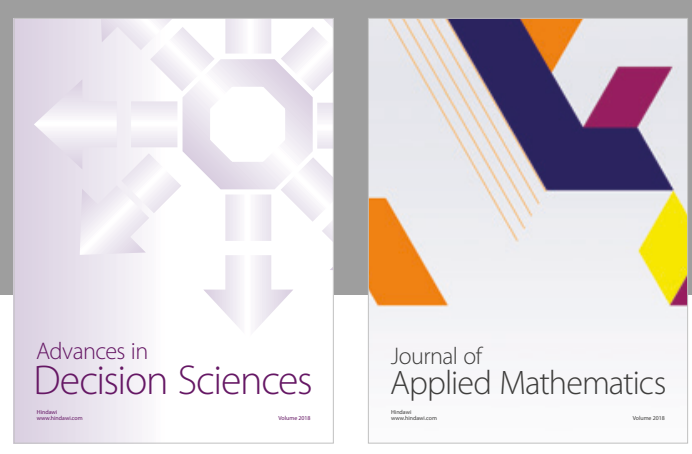

Journal of

Applied Mathematics
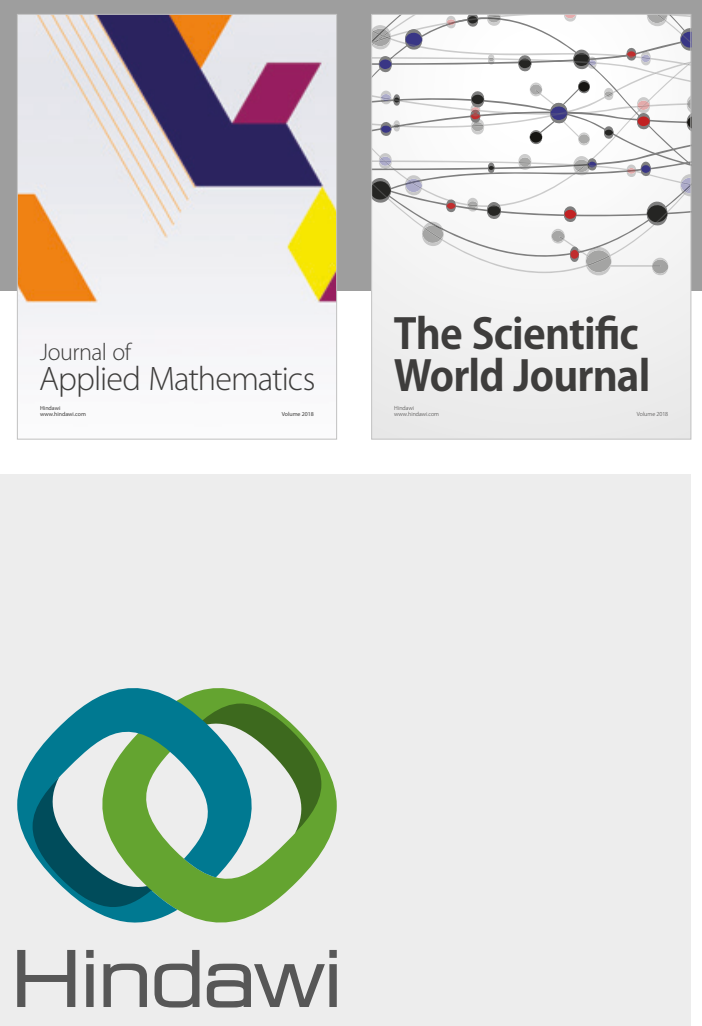

Submit your manuscripts at

www.hindawi.com

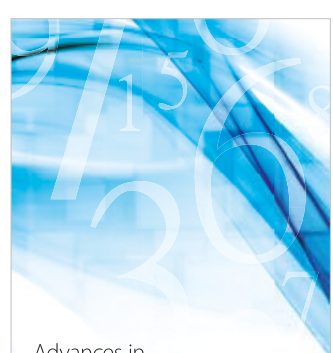

Advances in
Numerical Analysis
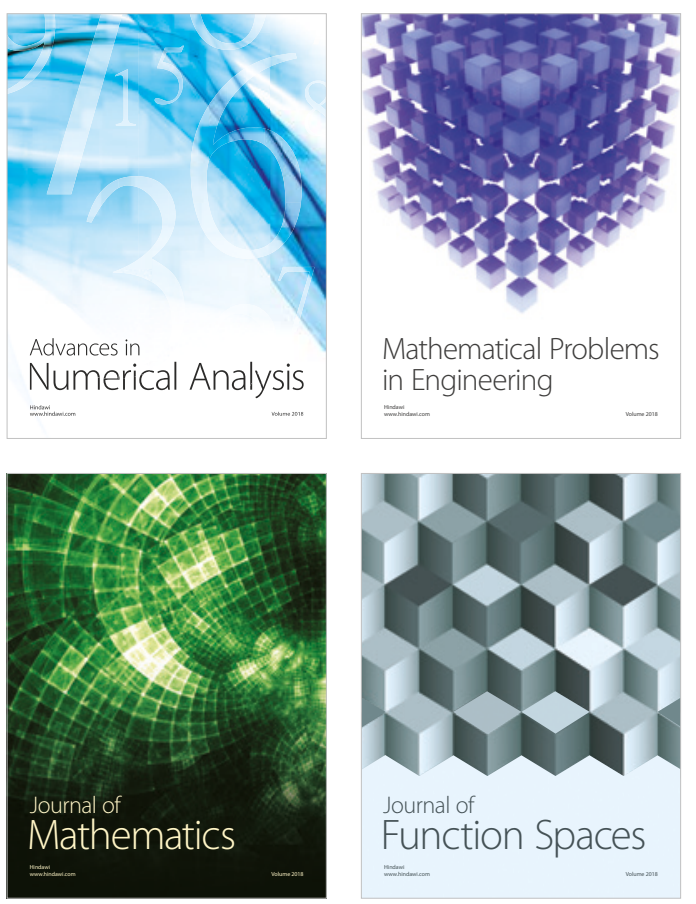

Mathematical Problems in Engineering

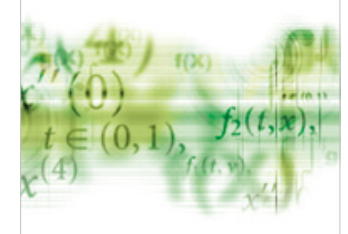

International Journal of

Differential Equations

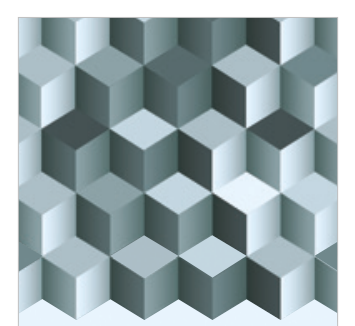

Journal of

Function Spaces
The Scientific

World Journal

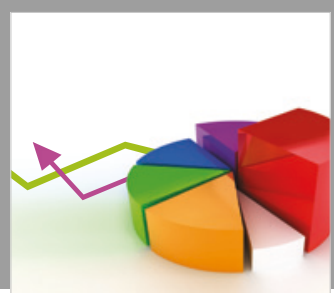

Journal of

Probability and Statistics
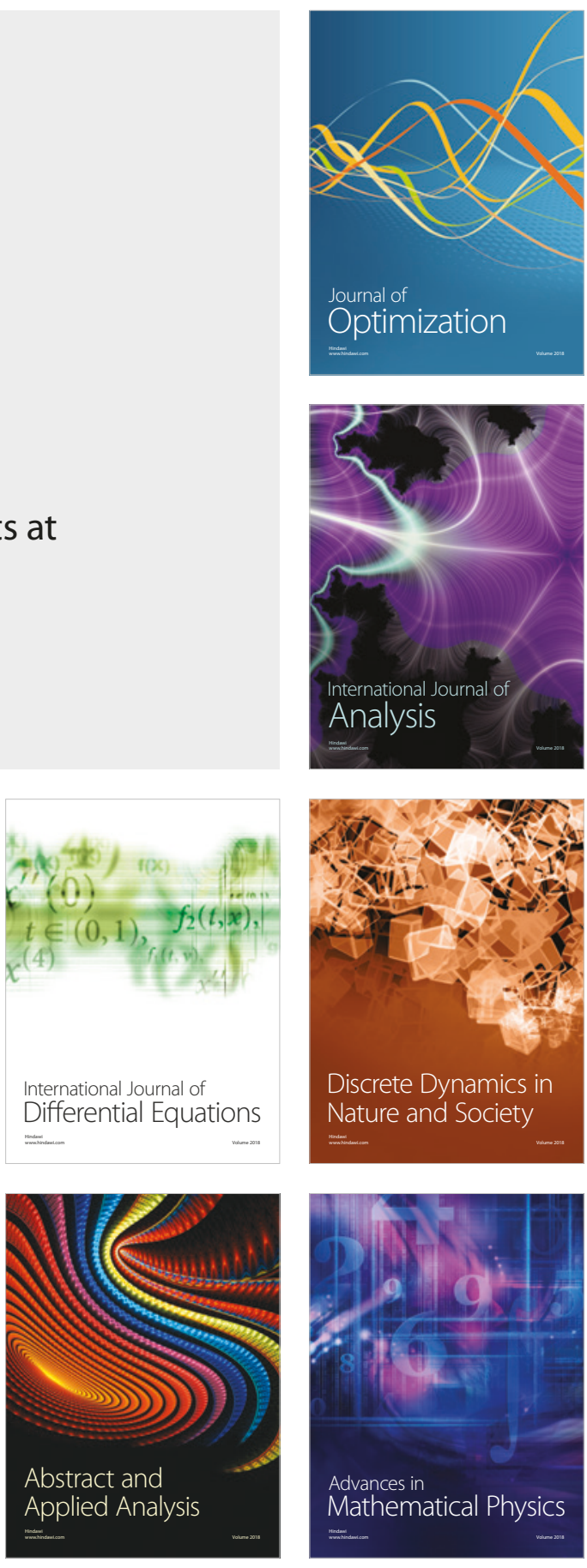